\title{
Synthesis and Characterization of Nickel-Alumina Composites from Recycled Nickel Powder
}

\author{
V. G. Karayannis ${ }^{1}$ and A. K. Moutsatsou ${ }^{2}$ \\ ${ }^{1}$ Department of Pollution Control Technologies, Technological Educational Institute (TEI) of West Macedonia, \\ Kila, 50100 Kozani, Greece \\ ${ }^{2}$ School of Chemical Engineering, National Technical University of Athens (NTUA), 9 Iroon Polytechniou Street, \\ 15773 Athens, Greece \\ Correspondence should be addressed to V. G. Karayannis, vkarayan@kozani.teikoz.gr \\ Received 5 December 2011; Revised 17 January 2012; Accepted 18 January 2012 \\ Academic Editor: Wen-Hua Sun
}

Copyright ( 2012 V. G. Karayannis and A. K. Moutsatsou. This is an open access article distributed under the Creative Commons Attribution License, which permits unrestricted use, distribution, and reproduction in any medium, provided the original work is properly cited.

\begin{abstract}
The recycling of metallic waste to create more valuable materials and their valorization into upgraded metal-based composites constitutes an important field of study. The composite industry nowadays considers environmental improvements as important as other properties of the materials. In the present paper, nickel powder was recycled from ferrous scrap, a low-cost and largely available material, by an effective hydrometallurgical recovery process. Then, this recycled powder was successfully used along with particulate $\alpha$-alumina to prepare oblong nickel-based composite specimens with ceramic reinforcement loadings ranging from 0 to $30 \mathrm{wt} . \%$ by applying powder processing manufacturing techniques including cold isostatic pressing (CIP) and sintering. The microstructures obtained were characterized, the specimens were subjected to three-point bend tests, and their fracture behaviour was evaluated. By increasing the \% ceramic reinforcement content, density clearly decreases while strengthening is achieved, thus leading to development of lightweight and enhanced oblong nickel-alumina composites. The composite microstructure, and particularly the metal-ceramic interface bonding, has a strong impact on fracture behaviour upon external loading.
\end{abstract}

\section{Introduction}

The valorization of materials recovered from scrap (metallic waste), a low-price secondary material, into value-added composites, represents a challenge with important technological, economic, and environmental aspects. Metalceramic composites, in particular, often exhibit improved properties with respect to their corresponding monolithic counterparts [1-7]. The development of such upgraded composites starting from materials recycled from metallic waste may be of particular interest, because of the possibility to reduce the production cost while also responding to environmental demands that are nowadays imposed by the decrease of ore deposits as well as by problems caused by waste rejection. Furthermore, including recyclates in products can lower carbon footprint and provide a marketing edge. It should be noted that the composite industry prioritizes nowadays environmental improvements as much as other composite properties and several companies are now incorporating recycled materials in composite products.

In order to recover high-grade ferrous and nonferrous metal fractions from metallic waste, much attention has been paid to separation processes for the various materials contained in scrap [8-10]. Usually, pyrometallurgical and hydrometallurgical processes are employed for treating solid wastes. High-energy requirement and need of dust collecting and gas cleaning systems are known as the major drawbacks of the pyrometallurgical methods. The hydrometallurgical processes are more environmentally suitable and economic to treat such materials and play an important role in the extraction and recovery of metals from various media. Besides the operational cost, leaching medium also decides what method of acid leaching will be suitable-relatively cheap acetic acid, cheap and available sulphuric acid, or aggressive chloride medium [11-14]. Therefore, an obvious 
opportunity for hydrometallurgical processing lies in recycling and processing of secondary sources such as ferrous scrap. However, hydrometallurgy has not yet found much application in this area, due to problems such as slow dissolution of the scrap and instability of highly concentrated solutions.

In the present study, the recovery of nickel powder from metallic waste (ferrous scrap), a low cost and largely available material, was attained by an attractive hydrometallurgical process $[15,16]$ that can provide metal and alloy powders, with properties generally similar to typical commercial grades, thus appropriate for the elaboration of nickel-based composites. The cost of the so-produced nickel powder has been estimated as less as $1 / 3$ of that of commercial grades.

For investigating the development of metal-based composites, nickel-alumina can be chosen as a model metalceramic system. Actually, nickel presents a great interest for various applications. Especially, powder metallurgical nickelalloy products show improved properties over conventional cast and wrought alloy products. Moreover, nickel-base alloys in the form of superalloys have already proven their usefulness in demanding environments [17-20]. Alumina has low density, electrical resistivity and coefficient of thermal expansion, high hardness, and specific stiffness, and is one of the most stable oxides, providing excellent oxidation and corrosion resistance and good high-temperature mechanical properties. In the form of embedded particles, coatings, or substrates, alumina is now an integral part of novel thermal barrier coating systems proposed for advanced applications [21-24]. Also, the properties of pure materials of this metal-ceramic system are well characterized in a wide temperature range. Indeed, there exists a large mismatch in properties between these two materials that poses a challenge for combining the attractive characteristics of each constitutive phase in a new optimized material with advanced behaviour. Moreover, such metal-ceramic materials, particulate or even functionally graded, can be formed through conventional and economical powder metallurgical routes, although chemical techniques, like in situ reaction processing or sol-gel processing, are also investigated for that purpose [25-30]. In addition, the microstructure stability of this system allows heating at the relatively elevated temperatures required for sintering without formation of undesirable brittle interfacial reaction products, given that nickel does not react with alumina under $1150^{\circ} \mathrm{C}$ [31]. In consequence, microstructures consisting of nickel and alumina are broadly considered able to satisfy the standards for structural applications in high-temperature demanding environments.

The encouraging results of a preliminary research using lower cost nickel powder recycled from ferrous scrap (by the aforementioned hydrometallurgical process) in nickelalumina pellets fabrication by uniaxial cold pressing, an elemental powder metallurgy technique, lead in the present study to the application of a more effective solid-state powder metallurgy method, comprising cold isostatic pressing (CIP) followed by sintering, in order to elaborate oblong nickelalumina composites starting from recycled nickel powder and particulate $\alpha$-alumina, and to evaluate their mechanical and fracture behaviour.
TABLE 1: Ferrous scrap characterization.

\begin{tabular}{lc}
\hline Elements & $\%$ \\
\hline $\mathrm{Fe}$ & 64.4 \\
$\mathrm{Cr}$ & 18.2 \\
$\mathrm{Ni}$ & 12.7 \\
$\mathrm{Mo}$ & 2.7 \\
$\mathrm{Mn}$ & 1.1 \\
$\mathrm{Si}$ & 0.5 \\
$\mathrm{Co}$ & 0.2 \\
$\mathrm{Cu}$ & 0.1 \\
$\mathrm{C}$ & 0.03 \\
$\mathrm{~S}$ & 0.003 \\
$\mathrm{P}$ & 0.007 \\
$\mathrm{Ti}$ & Traces \\
\hline
\end{tabular}

\section{Materials and Methods}

2.1. Raw Materials-Recycling. In Greece, the market research regarding ferrous scrap leads to the conclusion that only a small percentage of this solid waste is recycled in the steel production industries, covering a $50 \%$ of the required raw material, while stainless steel scrap is exported. Unfortunately, the main part of ferrous scrap is rejected raising increasing environmental concerns.

In the present study, samples of this secondary raw material in the form of industrial cutting disks from highspeed tools were selected. According to the analyses results presented in Table 1, this ferrous scrap can be classified as stainless steel 316, an alloy that is broadly used in various applications, from chemical industry to pipeline and reactor construction.

A hydrometallurgical process was developed, according to which, iron, chromium, nickel, and molybdenum can be recovered from ferrous scrap in powder form, appropriate for powder metallurgy applications. A hydrometallurgical process consists in general of a dissolution stage, a solvent extraction, and the stripping of loaded organic solution stage, and finally the stage of condensation and crystallization of the metal salts. Basic objectives of the recovery process were the following:

(i) extraction of metals of the highest economic value,

(ii) use of simple unitary operations requiring a minimum investment,

(iii) working at low temperature with dilute reagents and at low concentrations,

(iv) the working liquor should be recyclable to minimize costs,

(v) production of a final product in powder form with considerable added value.

Dissolution of scrap was performed by leaching with hydrochloric acid loaded with chlorine gas. After evaluation, leaching temperature and time conditions employed were $60^{\circ} \mathrm{C}$ and $1.5 \mathrm{~h}$, respectively. Versatic 6 (Shell Company 
Ltd.), a synthetic carboxylic acid, and commercial Kerosene were used for the solvent extraction step. Versatic 6 is a dialkyl alkanoic acid of a molecular weight of 116 , empirical formula $\mathrm{C}_{6} \mathrm{H}_{12} \mathrm{O}_{2}$, and purity $99 \%$ (according to the technical bulletin of the company). Due to the utilization of this acidic cation exchanger, it is easy, by changing the solution $\mathrm{pH}$, to achieve selective retention of the metals by forming complexes. Mixing the scrap solution with the organic phase at $\mathrm{pH}=2$, molybdenum is being bound. After the separation of the phases by adjusting the aqueous phase to $\mathrm{pH}=3$, iron is separated, and repeating the procedure at $\mathrm{pH}=5.3$, chromium is drown away. At last, the aqueous solution is enriched in nickel, and then nickel chloride crystals are obtained by evaporation and crystallization. Finally, nickel powder is produced by hydrogen reduction of the nickel chlorides at $300^{\circ} \mathrm{C}$ for $1.5 \mathrm{~h}$. The flowsheet of the hydrometallurgical process for nickel recovery in powder form is presented in Figure 1, where the optimum proposed experimental conditions are also reported.

The nickel powder so-produced and then used as a raw material for the fabrication of nickel-based composites has a purity of $99.90 \%$, a specific gravity of $8.83 \mathrm{~g} / \mathrm{cm}^{3}$, and a specific surface of $3500 \mathrm{~cm}^{2} / \mathrm{g}$. Therefore, its properties are similar to these of typical commercially available powders produced by atomization. $\alpha$-Alumina powder (corundum, $0.9 \mu \mathrm{m}$ mean particle size, $99 \%$ purity) from Aldrich Chemical Company, Inc. was selected as the ceramic constituent. The particle size distribution of nickel and alumina powders used is comparatively depicted in Figure 2. A difference in granulometry between metal and ceramic powders is shown in Figure 2. Such particle size differences have been considered beneficial to more efficient particle packing during powder mixture compression.

2.2. Fabrication of Nickel-Alumina Composites. Powder processing techniques were applied in order to prepare nickelalumina composite samples with alumina loadings ranging between $0-30 \mathrm{wt} . \%$. First, nickel and alumina powders were dry mixed, and the mixtures were pressed to form a series of oblong cylindrical composite bars, appropriate for mechanical testing, by using cold isostatic pressing (CIP, 2000 bar). Compaction difficulties with regard to composite specimen integrity and strength, especially in the specimens with the higher $\%$ alumina amount, were observed. To overcome these hindrances, polypropylene carbonate (PPC) was incorporated in all mixtures as a binder. Then, the sintering procedure was carried out within a temperature/atmosphere controlled furnace for the consolidation of the composite bars. The following sintering conditions were selected after optimization: all compacts were first preheated at $400^{\circ} \mathrm{C}$ for $1 \mathrm{~h}$ for the additive decomposition, then sintered at $1200^{\circ} \mathrm{C}$ for $4 \mathrm{~h}$ in inert atmosphere, and finally gradually cooled to room temperature in the furnace, also in inert atmosphere, to minimize oxidation and quenching.

2.3. Characterization and Mechanical Testing. Phase identification of the sintered specimens was conducted by X-ray diffraction (XRD) measurements (Siemens, Diffractometer
D-5000). The microstructural characterization of the samples was realised by light optical microscopy (Olympus, BX60M) as well as by Scanning Electron Microscopy (SEM) (Jeol, JSM-6400) coupled with Energy Dispersive $\mathrm{X}$-ray Spectroscopy (EDX) analysis. Apparent density after sintering was measured by using a specific apparatus (Shimadzu, SMK401-AUW220V) according to the water immersion (Archimedes') method. Then, relative densities were calculated using theoretical densities of the individual constituents. Modulus of elasticity and fracture strength were determined from three-point bending tests carried out on rectangular bars-machined from the fabricated cylindrical ones and then ground and polished on the tensile surface-in a load frame (Instron), with a crosshead speed of $0.1 \mathrm{~mm} / \mathrm{min}$. The initial portion of the loading curve was used for the Young's modulus determination. All samples were loaded to failure, except for the $100 \%$ nickel ones for which the plastic deformation exceeded $2 \%$. Finally, fracture surfaces were studied.

\section{Results and Discussion}

After sintering, densified nickel-alumina oblong composite specimens were successfully developed (Figure 3 ).

The high purity and the relatively small particle size (providing an increased specific surface energy) of the used nickel powder recycled from metallic waste should contribute to an acceptable consolidation degree obtained at the relatively moderate sintering temperatures applied in the present work, as it can be deduced from the optical micrographs of sintered 20 and $30 \mathrm{wt} . \%$ ceramic loading nickel-alumina composites shown in Figure 4. In these micrographs, lighter phase represents the metal and darker the ceramic constituent.

At the sintering temperatures selected, densification occurs by coalescence of nickel particles by atomic diffusion that forms typical solid-state sintering necks binding the metal-ceramic composites, while poorer connectivity can be suggested in the ceramic phase. At lower \% ceramic addition (Figure 4(a)), the volume part of nickel phase containing interconnected metal particles apparently prevails over the ceramic part, thus a microstructure of a nickel matrix reinforced with alumina is obtained. However, when increasing the $\%$ ceramic constituent (Figure $4(\mathrm{~b})$ ), metal contiguity is decreased and the volume of sintered metal parts isolated between the ceramic additions is more pronounced.

Metal (nickel) and ceramic (alumina) phases remain separate in Figure 4, an indication of limited interdiffusion. A recent study attempted to elucidate the microscopic nature of metal-ceramic bonding and the influence of oxygen vacancies on adhesion at such metal/ceramic interfaces, and particularly at nickel/zirconia nickel-iron/zirconia interfaces, concluding that bonding mechanisms can involve two types of interactions, namely, the nickel-zirconium hybridization and nickel-oxygen ionic bonding [32].

From Figure 4, agglomerates of 5-25 $\mu \mathrm{m}$ are revealed in the ceramic phase. The particle size difference between metal and ceramic powders used (see Figure 2), although being 


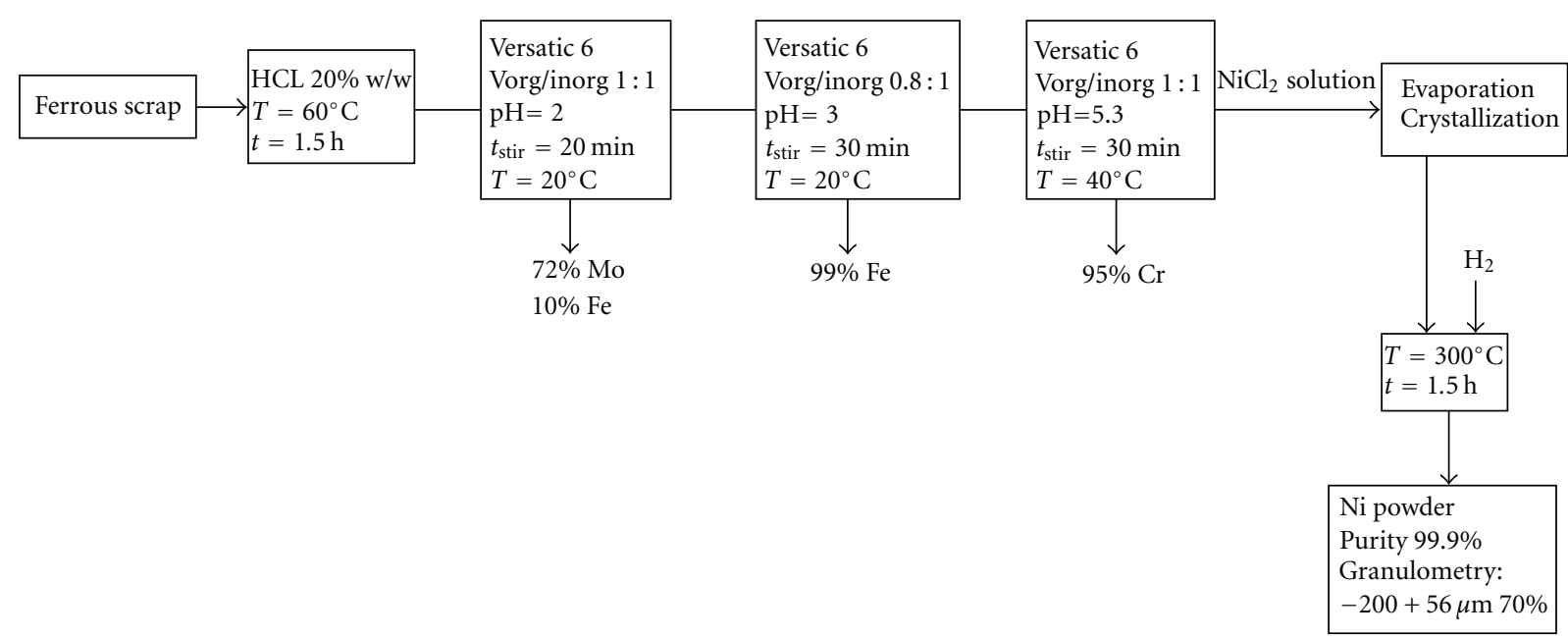

FIGURE 1: Flowsheet of the hydrometallurgical method for nickel recovery in powder form, showing the process optimal conditions.

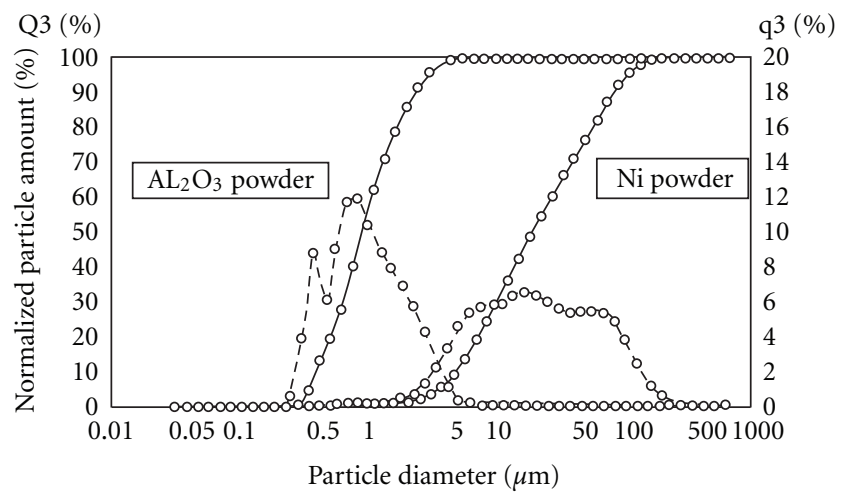

FIGURE 2: Particle size distribution of nickel and alumina powders.

considered beneficial to consolidation degree, may, at least partly, be responsible for the formation of such ceramic clusters when using powder compaction procedures.

Alumina discrete particles or agglomerates, not always uniformly distributed in the nickel matrix, are located in Figure 5 that provides SEM micrographs of a sintered $20 \mathrm{wt} . \%$ ceramic loading nickel-alumina composite, at two magnifications (the darker phase represents nickel and the lighter alumina, according to EDX analysis results). Also, an interfacial porosity is clearly revealed. These results lead to the conclusion that the necessary compression additive occupied a significant volume in these materials, thus creating pores after its removal during the green compacts thermal treatment. A bulk porosity ranging from $13.7 \%$ to $35.9 \%$ when increasing the $\%$ ceramic loading from 0 to $30 \mathrm{wt} . \%$ was determined in the sintered specimens. For this variation in consolidation degree, matrix volume contiguity limitations with the increasing \% alumina addition should be taken into consideration.

Apparent density of the sintered samples decreases from $7.6 \mathrm{~g} / \mathrm{cm}^{3}$ to $4.1 \mathrm{~g} / \mathrm{cm}^{3}$ when increasing the $\%$ amount of alumina introduced in the composites from 0 to $30 \mathrm{wt} . \%$,
FIGURE 3: Photograph of a sintered 30 wt. $\%$ ceramic content nickelalumina composite.

due to the lower density of the ceramic versus the metallic constituent. The obtained results are even lower than the density values theoretically expected according to the rule of mixtures, because of residual porosity, thus confirming the microstructural observation results.

Fracture behaviour of the specimens is illustrated in Figure 6 that shows fracture surfaces of sintered nickelbased composite specimens after mechanical testing. In these micrographs, lighter phase depicts alumina and darker nickel matrix.

Deflection of catastrophic cracks from their initial propagation front in the nickel matrix, which is observed in Figures 6(a), 6(b), and 6(c) and is believed to have beneficial effects on mechanical performance, can be associated with dislocations that are generated in the composites more ductile parts and can move during external loading to allow matrix plastic deformation, thus resulting in this effective mechanism 


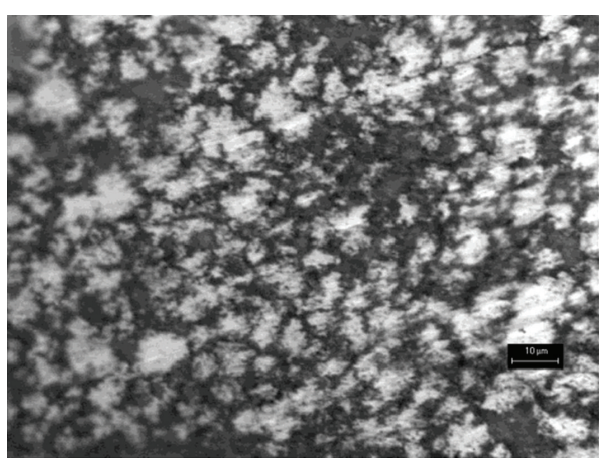

(a)

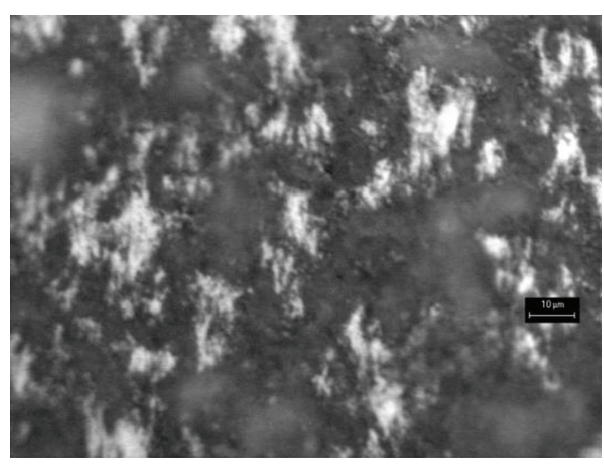

(b)

Figure 4: Micrographs of sintered $20 \mathrm{wt} . \%$ (a) and $30 \mathrm{wt} . \%$ (b) ceramic content nickel-alumina composites.

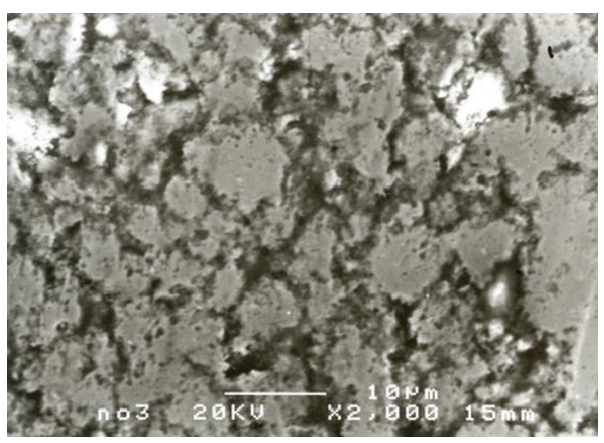

(a)

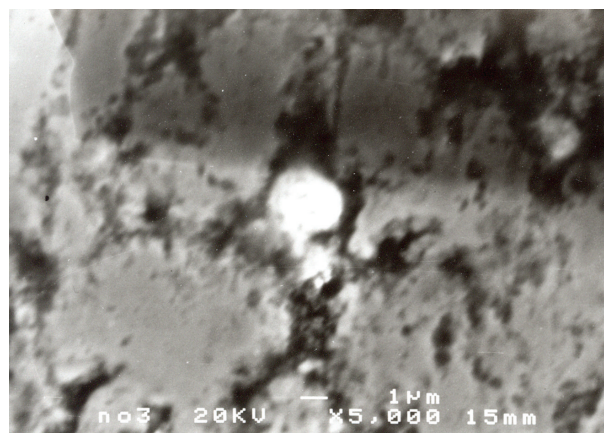

(b)

FIGURE 5: Micrographs of a sintered $20 \mathrm{wt}$ \% ceramic content nickel-alumina composite, at two magnifications.

of crack deflection. Such dislocations are due to residual thermal tensile stresses generated along the nickel-alumina interface during the composites cooling from the sintering temperature, because of a large thermal expansion coefficient mismatch between the constituents. In fact, in the ductile areas of the composites exhibiting strong interfacial bonds, relative interfacial movement of metal and ceramic materials as a result of these stresses is impeded, and therefore a matrix is locally deformed along the interface by the creation of high dislocation density. It should be emphasized here that this deflection of cracks preexisting or developed during external loading, by preventing an easy and continuous propagation due to the insertion of stiffer particulate reinforcement such as alumina, is precisely one of the main reasons to incorporate ceramic particles in a metal matrix. Crack propagation deflection in the matrix would possibly be even more intense if an interconnected ceramic network was also formed that could lead to stronger interfacial bonding. Frequent crack propagation through the ductile metal phase, such as this revealed in Figures 6(a), 6(b), and 6(c), must be responsible for some roughness the fracture surfaces exhibit, as a result of a certain degree of plastic deformation of nickel grains with necking before fracture occurs.

On the other hand, a weak interface in composites brittle areas-where a limited plasticity exists and strain distribution is mainly controlled by relative thermal expansion coefficient and modulus of the constituentsallows some interfacial sliding as a result of residual thermal stresses. However, such interfacial movement is nonuniformly restricted by reinforcement network, thus leading in greater plastic deformation in some areas, and possibly even in the development of microcracks, mainly at the interface. These phenomena could be even more pronounced if higher fabrication temperatures were applied resulting in more intense thermal stresses in the bulk of the materials. Actually, the experimental evidence showed (Figure 6(d)) that, in case of such a weak interface, plastic deformation during loading is followed by microcrack deflection along the metal-ceramic interface with its partial debonding that leads to a rather irregular crack propagation path. Such deflection, indicative of nonelastic actions taking place at the interface, as a result of the weak in this case interfacial bonding, is not able to act as a reinforcement mechanism, hence load transfer from matrix to ceramic reinforcement is decreased, thus degrading the composites strength. Naturally, crack deflection along the interface is preferable than crack propagation by ceramic particle cleavage or separation of contiguous particles in ceramic clusters. Such microdamages may develop during external loading or even originate from cooling. Microdamage accumulation can cause voids generation in the bulk of the materials, thus finally leading composites to failure. Actually, such brittle fracture of ceramic particles or clusters has also 


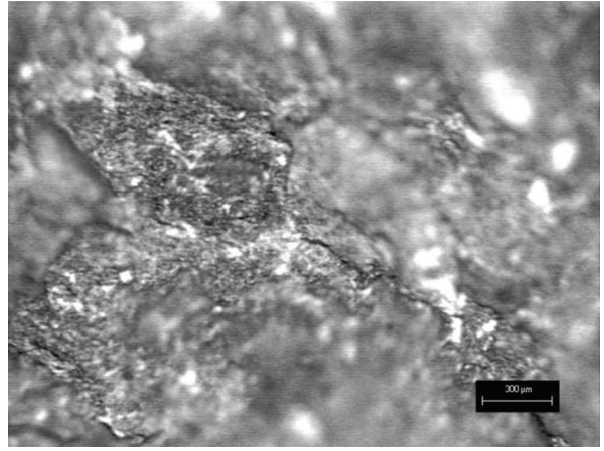

(a)

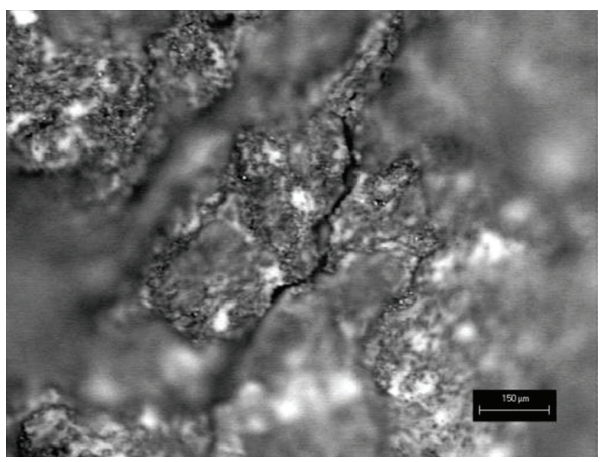

(c)

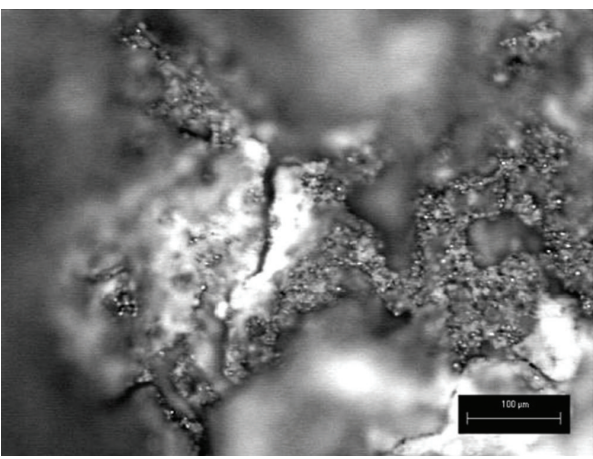

(e)

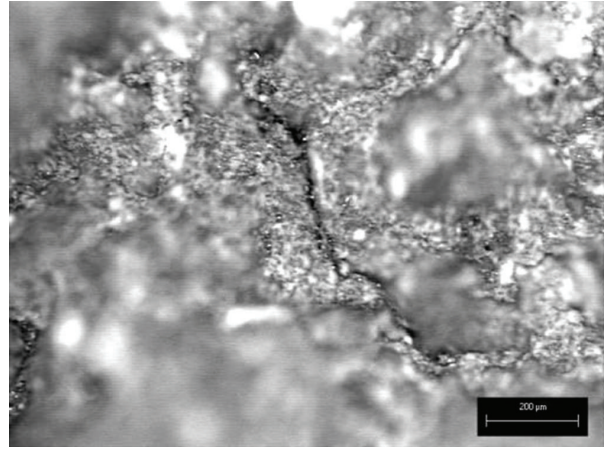

(b)

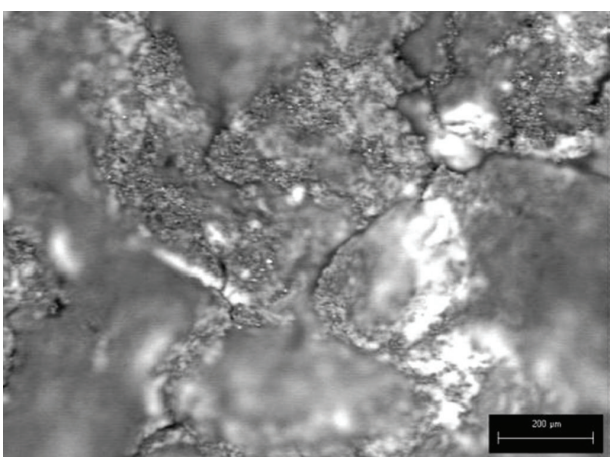

(d)

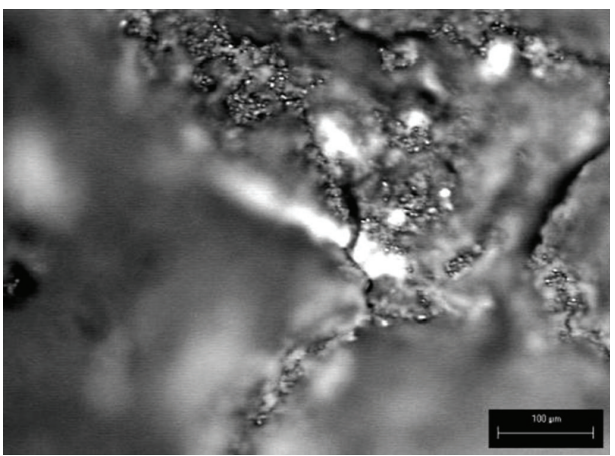

(f)

FIgURE 6: Micrographs of fracture surfaces of sintered nickel-alumina composites after mechanical testing.

been located in some areas of these materials (Figures 6(e) and $6(\mathrm{f}))$. So, it can be concluded that fracture of a rather mixed intergranular and transgranular type is obtained.

Crack initiation, propagation, and growth are not only influenced by metal-ceramic interfacial bonding and microdamage accumulation, but also by residual porosity. In particular, the interfacial porosity present in these materials causes matrix-ceramic particles decohesion under lower stresses and decreases the ability of load transfer to the reinforcement, and therefore it is detrimental to strength. Matrix porosity also affects the composites mechanical performance, as it decreases the composites load bearing area. In consequence, fracture occurs sooner after strain localization begins. Pore size distribution, pore shape, and flaws also count.
Lack of homogeneity of the reinforcement distribution in the nickel matrix may also have an impact on strength. Particle clusters observed in the ceramic phase can be considered as sites of inherent potential microdamage, as they are not able to support the same amount of stress as nonclustered particles, because matrix areas surrounding them are subjected to higher stresses. Therefore, stress concentration regions are introduced in the matrix, hindering its plastic flow. Hence, matrix strengthening is restricted, as the development and accumulation of internal microdamages during testing is more likely to occur at lower applied stresses.

All damages existing in the bulk of the materials, in the form of either debonding between metal and ceramic phases or poor sintering between contiguous ceramic particles or particle fracture, have even been treated in other studies 


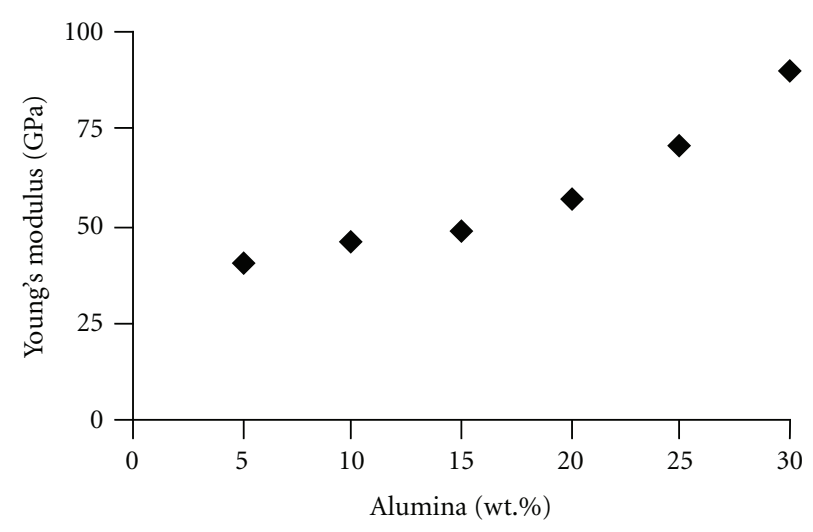

(a)

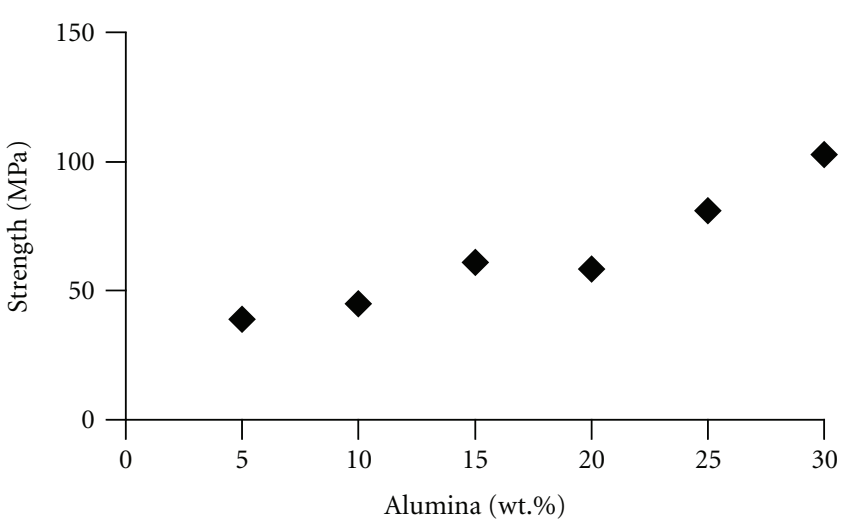

(b)

FIGURE 7: Young's modulus (a) and fracture strength (b) of nickel-alumina composites as a function of ceramic content (wt.\%).

on nickel-alumina composites as porosity deteriorating mechanical performance [33].

The mechanical properties values of the nickel-based composites versus the alumina content (wt.\%) are plotted in Figure 7.

Young's modulus and flexural strength of the composites increase progressively when increasing the addition of stiffer alumina particles. It can be suggested that this strengthening was achieved by two types of mechanisms: firstly, straininduced load transfer from nickel matrix to reinforcement, given the relatively high \% particulate ceramic loading, although matrix strength maintains an important role; secondly, strengthening mechanisms from the ceramic particles effect on the nickel matrix deformation by the inhibition of dislocations motion, already discussed. The restriction of matrix grain growth due to reinforcement particles presence in the matrix, and even due to residual porosity, must also be taken into account. Moreover, the small particle size of the alumina used in the present study is considered beneficial to this reduction of matrix grain (by limiting the motion of grain boundaries) and even to the restriction of microdamages accumulation during mechanical testing. The relatively moderate sintering conditions selected after optimization also contributed to prevent an exceeding matrix grain growth and permitted avoiding formation of brittle interfacial reaction products, which, otherwise, could lead to a weaker interface bond.

This increase in mechanical properties (Figure 7) combined with the aforementioned clear decrease in density leads to improvement of specific modulus and strength, which is broadly considered as one of the main advantages of metal-ceramic composites of this category. However, this improvement of mechanical performance is relatively limited, mainly because of the residual porosity revealed in these materials and the other microstructural features already discussed. Actually, porosity has a significant impact on mechanical behaviour, and a number of relationships have been proposed that relate the porosity level to mechanical properties. The empirical formulation (1) presented by Coble and Kingery has been used [25, 33] to correlate the elastic modulus, $E$, of nickel-alumina composites to the amount of porosity present in the composite, as follows:

$$
E=E_{0}\left(1-1.9 \rho+0.9 \rho^{2}\right)
$$

where $\rho$ is the porosity and $E_{0}$ is the Young's modulus at full density.

Bending ductility, in terms of minimum bend radius, the smallest radius around which a specimen can be bent without cracks being observed in the outer tension surface, varies slightly with the increasing ceramic loading. Naturally, it is clearly degraded compared to this of unreinforced nickel.

\section{Conclusions}

Nickel was recycled in powder form from metallic waste (ferrous scrap) by an effective hydrometallurgical process and successfully used into nickel-based composites with particulate alumina reinforcement ranging from 0 to $30 \mathrm{wt} . \%$. The high purity and the relatively small particle size of this nickel powder seems contribute to an acceptable consolidation degree at the relatively moderate sintering temperatures applied, rendering the production of oblong nickel-alumina composites feasible.

Mechanical behaviour depends on the \% reinforcement content. When increasing the $\%$ addition of stiffer ceramic particles, apparent density clearly decreases while strengthening is progressively achieved. The composite microstructure has a strong impact on fracture behaviour upon external loading. Particularly, microcrack initiation, propagation, and growth are influenced by metal-ceramic interfacial bonding as well as by microdamage accumulation originated from cooling or developed during loading. Microcrack propagation deflection in the nickel matrix, indicative of a strong interface, is frequently observed in the composite ductile areas. Thus, lightweight nickel-based composites with improved specific modulus and fracture strength are obtained, which may be important for several applications. The energy savings that can be attained by such reduction of 
weight must be emphasized from the environmental point of view.

\section{References}

[1] W. Callister, Materials Science \& Engineering: An Introduction, John Wiley \& Sons, New York, NY, USA, 7th edition, 2007.

[2] T. Clyne and P. Withers, An Introduction to Metal Matrix Composites, Cambridge University Press, Cambridge, UK, 1993.

[3] E. Marin, M. Lekka, F. Andreatta et al., "Electrochemical behaviour of Aluminum-fly ash composites prepared by powder metallurgy technique," in Proceedings of the 4rd World of Coal Ash Conference (WOCA '11), pp. 1-16, Denver, Colo, USA, 2011.

[4] U. Martin, D. Ehinger, L. Krüger et al., "Cellular energy absorbing TRIP-Steel/Mg-PSZ composite: honeycomb structures fabricated by a new extrusion powder technology," Advances in Materials Science and Engineering, vol. 2010, Article ID 269537, 6 pages, 2010.

[5] A. Moutsatsou, G. Itskos, P. Vounatsos, N. Koukouzas, and C. Vasilatos, "Microstructural characterization of PM-Al and PM-Al/Si composites reinforced with lignite fly ash," Materials Science and Engineering A, vol. 527, no. 18-19, pp. 4788-4795, 2010.

[6] N. Gupta, "Symposium review: processing, properties and performance of composite materials," JOM-Journal of the Minerals Metals and Materials Society, vol. 61, no. 3, pp. 4244, 2009.

[7] K. Kondoh, J. Umeda, and K. Kawabata, “Tribological analysis of $\mathrm{Mg}_{2} \mathrm{Si}$ particulates reinforced powder metallurgy magnesium alloy composites under oil lubrication condition," Advances in Materials Science and Engineering, vol. 2009, Article ID 628737, 8 pages, 2009.

[8] A. Agrawal and K. K. Sahu, "Problems, prospects and current trends of copper recycling in India: an overview," Resources, Conservation and Recycling, vol. 54, no. 7, pp. 401-416, 2010.

[9] J. Cui and L. Zhang, "Metallurgical recovery of metals from electronic waste: a review," Journal of Hazardous Materials, vol. 158, no. 2-3, pp. 228-256, 2008.

[10] K. K. Sahu, A. Agrawal, and B. D. Pandey, "Recent trends and current practices for secondary processing of zinc and lead. Part II: zinc recovery from secondary sources," Waste Management and Research, vol. 22, no. 4, pp. 248-254, 2004.

[11] Y. F. Shen, W. Y. Xue, and W. Y. Niu, "Recovery of Co(II) and $\mathrm{Ni}(\mathrm{II})$ from hydrochloric acid solution of alloy scrap," Transactions of Nonferrous Metals Society of China, vol. 18, no. 5, pp. 1262-1268, 2008.

[12] J. Avraamides, G. Senanayake, and R. Clegg, "Sulfur dioxide leaching of spent zinc-carbon-battery scrap," Journal of Power Sources, vol. 159, no. 2, pp. 1488-1493, 2006.

[13] B. Cohen, D. S. Shipley, A. R. Tong, S. J. G. Casaroli, and J. G. Petrie, "Precipitation of iron from concentrated chloride solutions: literature observations, challenges and preliminary experimental results," Minerals Engineering, vol. 18, no. 13-14, pp. 1344-1347, 2005.

[14] F. Habashi, "A short history of hydrometallurgy," Hydrometallurgy, vol. 79, no. 1-2, pp. 15-22, 2005.

[15] A. Moutsatsou, V. Karayannis, and C. Sotiriou, "Optimization of ferrous scrap dissolution for the recovery of $\mathrm{Fe}, \mathrm{Ni}, \mathrm{Cr}$ and
Mo powders by a hydrometallurgical process," in Proceedings of the 3rd International Symposium on HydrometallurgyHydromet, pp. 1-18, Falmouth, UK, 2007.

[16] V. G. Karayannis and A. Moutsatsou, "Ferrous scrap yields powders for PM \& MMCs," Metal Powder Report, vol. 55, no. 12, pp. 33-36, 2000.

[17] Q.-X. Tang, S. Ukai, A. Minami, and S. Hayashi, “ $y$ ' precipitation and growth kinetics in mechanically alloyed NiAl," Advances in Materials Science and Engineering, vol. 2011, Article ID 137387, 7 pages, 2011.

[18] C. G. Hjorth, "HIP powder metal near-net shapes for demanding environment and applications," Journal of Iron and Steel Research International, vol. 14, no. 5, pp. 121-125, 2007.

[19] B. A. Pint, J. R. DiStefano, and I. G. Wright, "Oxidation resistance: one barrier to moving beyond Ni-base superalloys," Materials Science and Engineering A, vol. 415, no. 1-2, pp. 255263, 2006.

[20] M. Lekka, N. Kouloumbi, M. Gajo, and P. L. Bonora, "Corrosion and wear resistant electrodeposited composite coatings," Electrochimica Acta, vol. 50, no. 23, pp. 4551-4556, 2005.

[21] A. G. Evans, D. R. Clarke, and C. G. Levi, "The influence of oxides on the performance of advanced gas turbines," Journal of the European Ceramic Society, vol. 28, no. 7, pp. 1405-1419, 2008.

[22] C. Fourmond, G. Da Rold, F. Rousseau et al., "Characterisation of thermal barrier coatings and ultra high temperature composites deposited in a low pressure plasma reactor," Journal of the European Ceramic Society, vol. 31, no. 13, pp. 2295-2302, 2011.

[23] C. Lynch, Ed., CRC Handbook of Materials Science, CRC Press, Boca Raton, Fla, USA, 1974.

[24] G. Shanmugavelayutham and A. Kobayashi, "Mechanical properties and oxidation behaviour of plasma sprayed functionally graded zirconia-alumina thermal barrier coatings," Materials Chemistry and Physics, vol. 103, no. 2-3, pp. 283289, 2007.

[25] D. E. Aldrich, Z. Fan, and P. Mummery, "Processing, microstructure, and physical properties of interpenetrating $\mathrm{Al}_{2} \mathrm{O}_{3} / \mathrm{Ni}$ composites," Materials Science and Technology, vol. 16, no. 7-8, pp. 747-752, 2000.

[26] A. E. Castro Luna and M. E. Iriarte, "Carbon dioxide reforming of methane over a metal modified $\mathrm{Ni}-\mathrm{Al}_{2} \mathrm{O}_{3}$ catalyst," Applied Catalysis A, vol. 343, no. 1-2, pp. 10-15, 2008.

[27] Q. Feng, T. Li, H. Teng et al., "Investigation on the corrosion and oxidation resistance of $\mathrm{Ni}-\mathrm{Al}_{2} \mathrm{O}_{3}$ nano-composite coatings prepared by sediment co-deposition," Surface and Coatings Technology, vol. 202, no. 17, pp. 4137-4144, 2008.

[28] S. C. Kuiry, S. Wannaparhun, N. B. Dahotre, and S. Seal, "In-situ formation of Ni-alumina nanocomposite during laser processing," Scripta Materialia, vol. 50, no. 9, pp. 1237-1240, 2004.

[29] J. Li, F. Li, and K. Hu, "Preparation of $\mathrm{Ni} / \mathrm{Al}_{2} \mathrm{O}_{3}$ nanocomposite powder by high-energy ball milling and subsequent heat treatment," Journal of Materials Processing Technology, vol. 147, no. 2, pp. 236-240, 2004.

[30] Q. Yan, G. Y. Wang, Z. Huang, and D. Jiang, "A microstructure study of $\mathrm{Ni} / \mathrm{Al}_{2} \mathrm{O}_{3}$ composite ceramics," Journal of Alloys and Compounds, vol. 467, no. 1-2, pp. 438-443, 2009.

[31] P. Lourdin, D. Juvé, and D. Tréheux, "Nickel-alumina bonds: mechanical properties related to interfacial chemistry," Journal 
of the European Ceramic Society, vol. 16, no. 7, pp. 745-752, 1996.

[32] S. V. Eremeev, S. Schmauder, S. Hocker, and S. E. Kulkova, "Ab-initio investigation of $\mathrm{Ni}(\mathrm{Fe}) / \mathrm{ZrO}_{2}\left(\begin{array}{lll}0 & 0 & 1\end{array}\right)$ and $\mathrm{Ni}$ $\mathrm{Fe} / \mathrm{ZrO}_{2}\left(\begin{array}{lll}0 & 0 & 1\end{array}\right)$ interfaces," Surface Science, vol. 603, no. 14, pp. 2218-2225, 2009.

[33] H. A. Bruck and B. H. Rabin, "Evaluating microstructural and damage effects in rule-of-mixtures predictions of the mechanical properties of $\mathrm{Ni}-\mathrm{Al}_{2} \mathrm{O}_{3}$ composites," Journal of Materials Science, vol. 34, no. 9, pp. 2241-2251, 1999. 

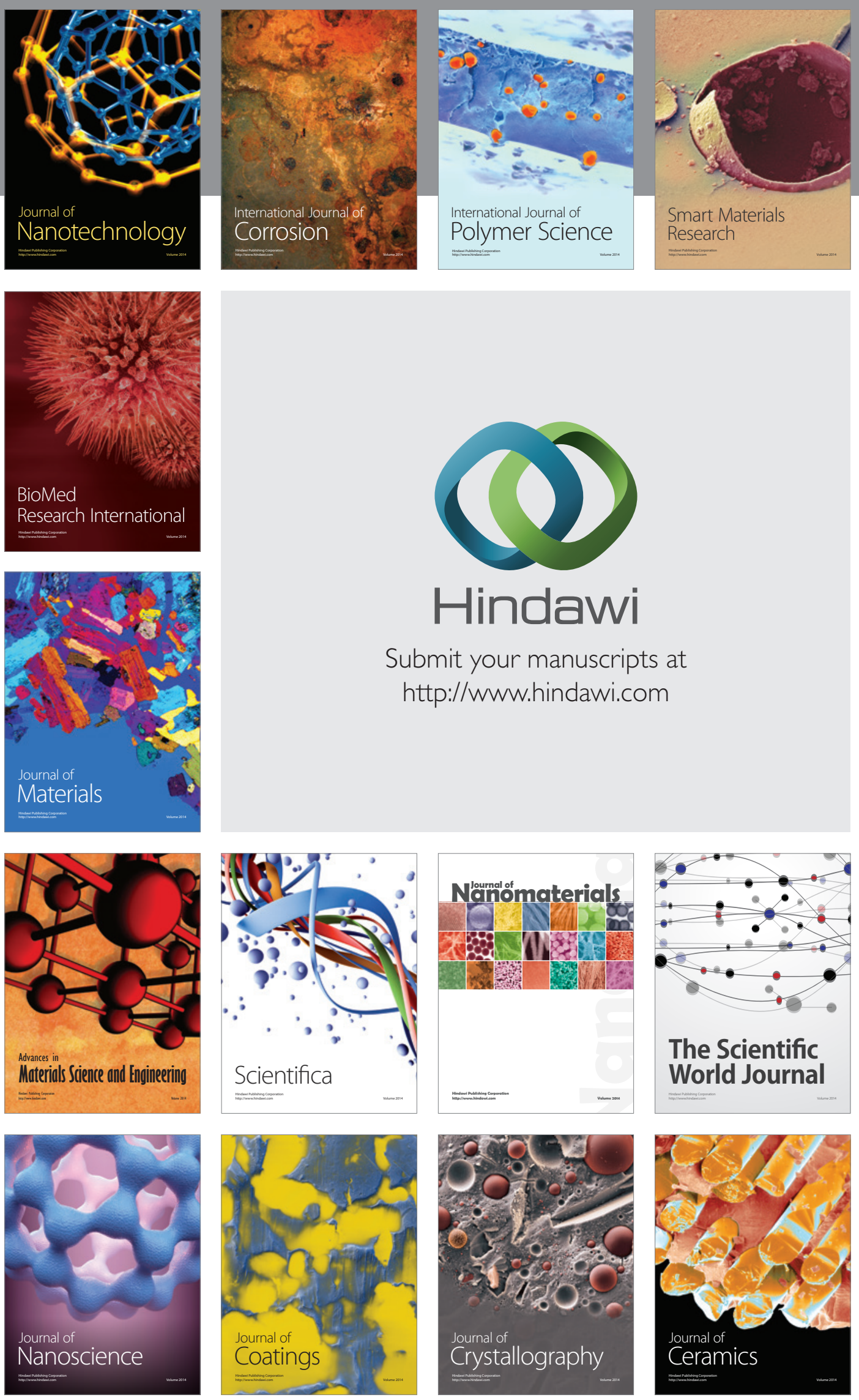

The Scientific World Journal

Submit your manuscripts at

http://www.hindawi.com

\section{World Journal}

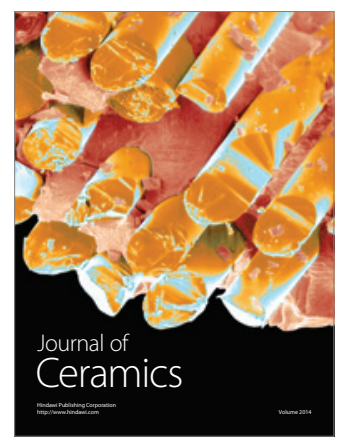

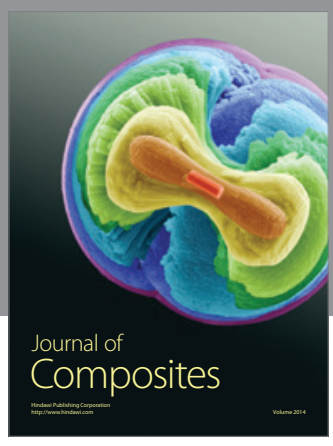
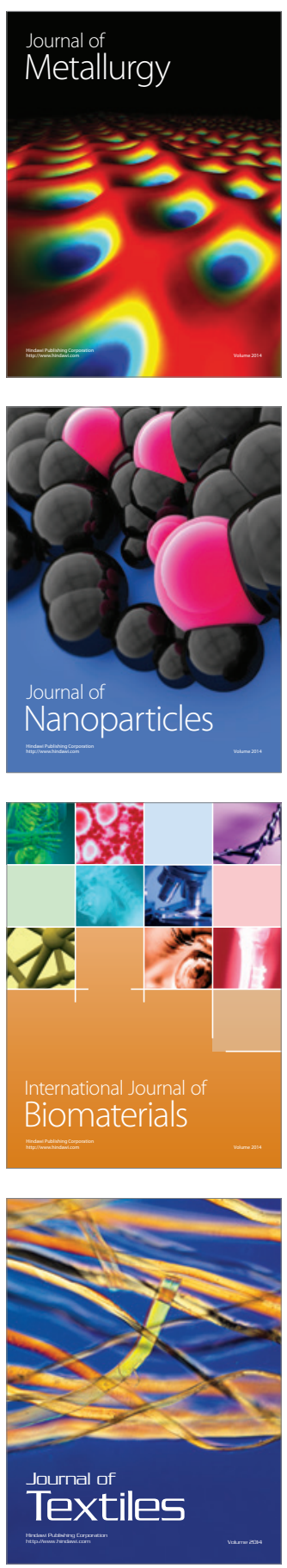